\title{
Delayed intrathyroidal hematoma causing respiratory distress after a seemingly benign fall: a case report
}

\author{
Lucas B. Chartier · Joel P. Turner
}

Received: 27 March 2010 /Accepted: 30 May 2010/Published online: 8 July 2010

(C) Springer-Verlag London Ltd 2010

\begin{abstract}
A rare event of a fall causing delayed intrathyroidal hematoma and respiratory distress is reported here. A 75-year-old woman with symptoms of vertigo causing syncope and fall $24 \mathrm{~h}$ earlier was seen and discharged from our emergency department after an unremarkable physical exam and 6-h observation period. Within $3 \mathrm{~h}$ of discharge, the patient was transported back by Emergency Medical Services with an enlarging neck mass and subjective respiratory distress. CT scan demonstrated a large, expanding hematoma, and the patient underwent an emergency hemithyroidectomy. Hürtle cell adenoma was found on pathologic specimen examination. A review of the literature of similar cases is presented, emphasizing the notion that concurrent thyroid pathology is a risk factor for airway compromise after seemingly benign trauma and that airway compromise can present in a delayed fashion.
\end{abstract}

Keywords Trauma $\cdot$ Thyroid $\cdot$ Hematoma .

Respiratory distress

L. B. Chartier $(\bowtie)$

Division of Emergency Medicine, Department of Medicine, University of Toronto,

2075 Bayview Ave., Rm. C753,

Toronto, ON, Canada M4N 2M5

e-mail: lucas.chartier@utoronto.ca

\section{J. P. Turner}

Department of Emergency Medicine,

Sir Mortimer B. Davis Jewish General Hospital,

3755, Côte-Ste-Catherine Road, Room D-027,

Montreal, QC, Canada H3T 1E2

\section{Introduction}

Although hemorrhage into the thyroid gland is well recognized, massive bleeding into the thyroid gland after trauma is rare. Given the anatomical location of the thyroid gland and the potential for airway compromise secondary to external compression, this is an issue that emergency physicians (EPs) must not overlook. We report the case of a patient presenting to the emergency department (ED) with a spontaneously expanding neck hematoma, approximately $24 \mathrm{~h}$ after a seemingly benign fall.

\section{Case report}

Mrs. C, a 75-year-old female, had been experiencing 4 days of dizziness before sustaining an unwitnessed syncopal episode at home after getting up from bed. She called the Emergency Medical Services (EMS), which transported her to a local ED. Except for a mild bradyarrhythmia, the patient was stable and was transferred to our hospital ED for further evaluation. She was known for benign paroxysmal positional vertigo (BPPV), atrial fibrillation and hypertension. Her medications included warfarin, spironolactone and telmisartan. On further questioning, the patient remembered experiencing vertigo prior to the fall. In the $\mathrm{ED}$, she continued to complain of vertigo, nausea, mild headache and tinnitus. She had one episode of vomiting while in the ED, which was managed with $50 \mathrm{mg}$ of dimenhydrinate intravenously. The patient appeared in no apparent distress. Her vital signs included a blood pressure of 175/85, an irregular pulse of approximately 75 and an oxygen saturation of $96 \%$ with a respiratory rate of 14 . She was afebrile. Her physical exam, including a complete musculoskeletal exam, was unremarkable except for bilat- 
eral chronic pedal edema. Of note, there was no neck pain, tenderness or mass. An electrocardiogram revealed atrial fibrillation with a ventricular rate of 66 to 75 beats per minute. Laboratory tests revealed a hemoglobin of $142 \mathrm{~g} / \mathrm{l}$, a white blood cell count $10.3 \times 10^{9} / 1$, a platelet count of $185 \times 10^{9} / 1$ and a blood glucose of $8.6 \mathrm{mmol} / 1$ (all within normal limits). The international normalized ratio (INR) was subtherapeutic at 1.6. The patient was given a diagnosis of "fall secondary to BPPV," and was discharged home asymptomatic and in stable condition after a 6-h observation period. She was instructed to return to the ED if her symptoms persisted.

Within $3 \mathrm{~h}$ of discharge, the patient called EMS complaining of vomiting and of an enlarging neck mass. She was immediately transported back to our hospital ED where a new team of EPs assessed her. The patient stated that the mass had been enlarging for the past hour and that it was accompanied by a sensation of fullness in her neck, dysphagia and some mild respiratory difficulties. After obtaining a history of a trauma $24 \mathrm{~h}$ earlier, cervical spine immobilization was immediately initiated. The patient had a blood pressure of $150 / 75$, an irregular pulse of approximately 68 and an oxygen saturation of $99 \%$ on 41 of oxygen by nasal prong. She was noted to be in minimal respiratory distress, with a respiratory rate of 22. On examination, the patient was found to have a large swelling in the right anterior triangle of the neck, at the level of the thyroid gland. The trachea was displaced to the left, but the patient displayed no stridor. She also had tenderness in the region of the cervical paraspinal muscles. A bedside ultrasound performed by the EP confirmed a large fluid-filled structure adjacent to the thyroid gland. A provisional diagnosis of "neck hematoma causing potential airway compromise" was given. Computed tomography scan (CT) of the patient's head, neck and cervical spine was immediately ordered. Four units of fresh frozen plasma were ordered to correct the patient's anticoagulation. The ear, nose and throat specialists (ENT), already in the ED, were consulted and immediately assessed the patient.

The CT of the patient's head and cervical spine showed no fracture or abnormality. The $\mathrm{CT}$ of her neck revealed a large hematoma, centered in the right thyroid lobe measuring $7.2 \times 5.0 \times 6.9 \mathrm{~cm}$. The trachea was moderately deviated towards the left but without significant reduction in the cross-sectional area. While ENT was establishing a course of action, the patient complained of a subjective increase in respiratory distress in the ED. Mrs. C was immediately transferred to the operating room, successfully intubated and had her neck mass explored. A large hematoma resulting from the torn right infrathyroid artery was discovered. Hemostasis was established, and the patient finally underwent a right hemi-thyroidectomy. The patient did very well postoperatively and was discharged from the hospital 2 days later. She was doing well at her follow-up appointment 2 weeks later. Pathologic examination of the resected specimen revealed an intrathyroidal hematoma involving a Hürthle cell adenoma.

\section{Discussion}

Spontaneous hemorrhage in the thyroid gland, although not frequent, is not an uncommon phenomenon [1, 2]. Blunt traumatic etiologies, however, are much more rare. Reported cases include direct blunt trauma to the neck from a motor vehicle steering wheel [3], bicycle handlebars [4], secondary to airbag deployment [5] as well as a fall on the neck [6]. Rare cases of thyroid hemorrhage following indirect trauma (trauma near the neck but not directly to the thyroid gland) $[1,7]$, and excessive Valsalva maneuvers $[2,8]$ have also been described. The symptoms displayed by the patients ranged from a painless neck lump [2] to sudden respiratory collapse [8]. The time to presentation to a physician ranged from immediate $[2,3,8]$ to more than $24 \mathrm{~h}[6,7]$. All but one [8] survived. In seven of the eight cases, a concomitant thyroid pathology was either known or subsequently discovered on pathologic examination [1-3, 5-8].

Thyroid pathology seems to be an important risk factor in causing neck hematoma following blunt trauma. This was illustrated as early as in 1922 by Terry's experimental work, in which he showed that in pathological states, coughing or straining raises the patient's central venous pressure sufficiently to cause thyroid vessel bleeding [9]. It is believed that many thyroid pathologies, including adenomas, induce angiogenesis of immature, fragile blood vessels that are prone to rupture $[10,11]$. This vulnerability is compounded by the fact that blood flow increases in diseased states [12], potentially causing vessel rupture with major bleeding after seemingly benign trauma. One important contributing factor unique to the present case was the fact that the patient was taking warfarin for atrial fibrillation. While no single cause seems sufficient to account for Mrs. C's intrathyroidal hemorrhage, the combination of different factors might have been enough to account for her significant hematoma. These may include a possible trauma to the neck during her fall, the action of warfarin and the presence of a Hürthle cell adenoma.

In light of the diverse nature of presentations in patients with trauma-related intrathyroidal hemorrhages illustrated here, a few points are noteworthy. All patients with obvious direct trauma to the neck and thyroid gland require close observation with respect to possible airway compromise. However, in patients with known or suspected thyroid pathology, even minor trauma can pose a risk for delayed intrathyroidal hemorrhage. These patients may require a prolonged period of observation before being safely discharged from the ED. Given that the currently discussed 
case is only the ninth reported in the literature, it is difficult to suggest any specific duration for monitoring. Since very delayed complications may still occur, patients and/or their caregivers should receive, upon discharge, appropriate and detailed information regarding indications to return to the ED. Luckily, most hemorrhages into thyroid adenomas are usually gradual and generally respond to conservative treatment. As opposed to previous recommendations [7], we do not recommend serial neck circumference measurements. We believe that given the different compressive nature of soft tissue masses, this measure would be ineffective at detecting and preventing airway compromise before it is subjectively or objectively apparent.

\section{Conclusion}

We illustrated the case of a patient presenting with an intrathyroidal hemorrhage causing respiratory distress approximately $24 \mathrm{~h}$ after a seemingly benign fall. It is important to recognize the potential for such complications, especially in the presence of thyroid pathology. The greatest challenge for the emergency physician is to recognize this risk factor and to consider the possibility of delayed airway compromise when deciding on the final disposition of the patient.

Conflicts of Interest The authors have no financial or other conflicts of interest related to this submission.

\section{References}

1. Grace RH, Shilling JS (1969) Acute haemorrhage into the thyroid gland following trauma and causing respiratory distress. Brit $\mathrm{J}$ Surg 56:635-636

2. Ryan WJ (1942) Hemorrhage into the thyroid gland resulting from muscular effort. Ann Surg 115:469-471

3. Blaivas M, Hom DB, Younger JG (1999) Thyroid gland hematoma after blunt cervical trauma. Am J Emerg Med $17: 348-350$

4. Rupprecht H, Ruümenapf G, Braig H et al (1999) Acute bleeding caused by rupture of the thyroid gland following blunt neck trauma: case report. J Trauma 36:408-409

5. Hirshoren N, Hocwald E, Eliashar R (2004) Isolated traumatic thyroid hemorrhage secondary to air bag deployment. Otolaryngol Head Neck Surg 130:791-793

6. Oka Y, Nishijima J, Azuma T et al (2007) Blunt thyroid trauma with acute hemorrhage and respiratory distress. J Emerg Med 32:381-385

7. Behrends RL, Low RB (1987) Acute goiter hematoma following blunt neck trauma. Ann Emerg Med 16:1300-1301

8. Wendel A (1936) Fetal adenoma with hemorrhage. Am J Surg 31:372-375

9. Terry WI (1922) Radium emanations in exophtalmic goiter-blood vessels of adenomas of thyroid. JAMA 79:1-3

10. Sobrinho-Simões M, Máximo V, Vieira de Castro I et al (2005) Hürthle (oncocytic) cell tumors of thytroid: etiopathogenesis, diagnosis and clinical significance. Int J Surg Pathol 13:29-35

11. Phitayakorn R, McHenry CR (2006) Follicular and Hürthle cell carcinoma of the thyroid gland. Surg Oncol Clin N Am 15:603623

12. Fowler PBS (2000) Thyroid, blood flow and atheroma. Q J Med 93:477-485 